HortSCIENCE 25(3):346-348. 1990.

\title{
Isozyme Markers for Cultivar Identification in Guayule
}

\author{
A. Estilai, A. Hashemi, and J.G. Waines \\ Department of Botany and Plant Sciences, University of California, \\ Riverside, CA 92521
}

Additional index words. Parthenium argentatum, starch gel electrophoresis, phosphoglucoisomerase, malate dehydrogenase, shikimate dehydrogenase, natural rubber, breeding

\begin{abstract}
Leaf extracts of 500 plants from 47 guayule (Parthenium argentatum Gray) entries including AZ-101, Gila, Cal-3, Cal-6, and Cal-7 germplasms; 12 accessions from Mexico; and a diverse array of diploid, triploid, and tetraploid selections were analyzed for isozyme variation of 17 enzyme systems. Glutamate oxalacetate transaminase (GOT, EC 2.6.1.1), isocitrate dehydrogenase (IDH, EC 1.1.1.42), malate dehydrogenase (MDH, EC 1.1.1.37), phosphoglucoisomerase (PGI, EC 5.3.1.9), shikimate dehydrogenase (SKDH, EC 1.1.1.25), and triosephosphate isomerase (TPI, EC 5.3.1.1) produced sharp and well-resolved bands. With the exception of AZ-101 and Gila, intraand inter-accession polymorphisms were present for the above enzymes. Plants of AZ101 and Gila showed identical banding patterns for every enzyme, supporting the view that these two germplasms may be the apomictic progenies of a single selection. Isozyme variations within entries indicated that most of the available guayule germplasms and selections are heterogeneous. Differences between entries suggested that isozymes may provide useful markers for cultivar identification.
\end{abstract}

Guayule, a perennial desert species native to northcentral Mexico and southwestern Texas, is being developed as a renewable source of natural rubber. The United States is totally dependent on outside sources and spends nearly $\$ 1$ billion annually to import Hevea natural rubber from southeast Asia (Estilai et al., 1988). Although seven new germplasms have been developed and released (Estilai, 1985, 1986; Tysdal et al., 1983), progress has been slow in the development of high rubber-yielding cultivars, primarily because of the complex breeding system of the species. Guayule plants are either diploid $(2 \mathrm{n}=36)$ and sexual, or polyploid ( $2 \mathrm{n}=54,72$, and higher) and facultative apomicts (Bergner, 1946; Esau, 1944). The occurrence of B-chromosomes among diploids and polyploids (Bergner, 1946; Catcheside, 1950) and the presence of

Received for publication 27 Feb. 1989. Research supported in part by USDA Grant no. 86-CRCR2-2834 and USDA-CSRS Agreement no. 58-3159$7-12$ a sporophytic system of self-incompatibility at the diploid level (Estilai, 1984; Gerstel, 1950) further complicates genetic studies. The nonavailability of easily recognizable genetic markers is also a factor in slow progress. So far, only one morphological marker, purple flower color, has been identified and genetically analyzed at the diploid level (Estilai, 1984).

Isozyme polymorphism has been successfully used for demonstrating genetic variation, identifying interspecific hybrids, and fingerprinting cultivars (Cardy and Kannenberg, 1982; Chaparro et al., 1987; Ellstrand, 1984; Garvin, 1987). In guayule, isozyme polymorphisms were used to show that the Cal-3 sexual diploid germplasm is not a truebreeding selection (Zaiger et al., 1984).

Isozyme markers associated with traits such as rubber content, rubber quality, and rubber yield, are expected to facilitate guayule breeding. In addition, these markers should be of value in determining the degree of apomixis in different genotypes.

The objectives of this study were to examine a wide array of guayule materials for 

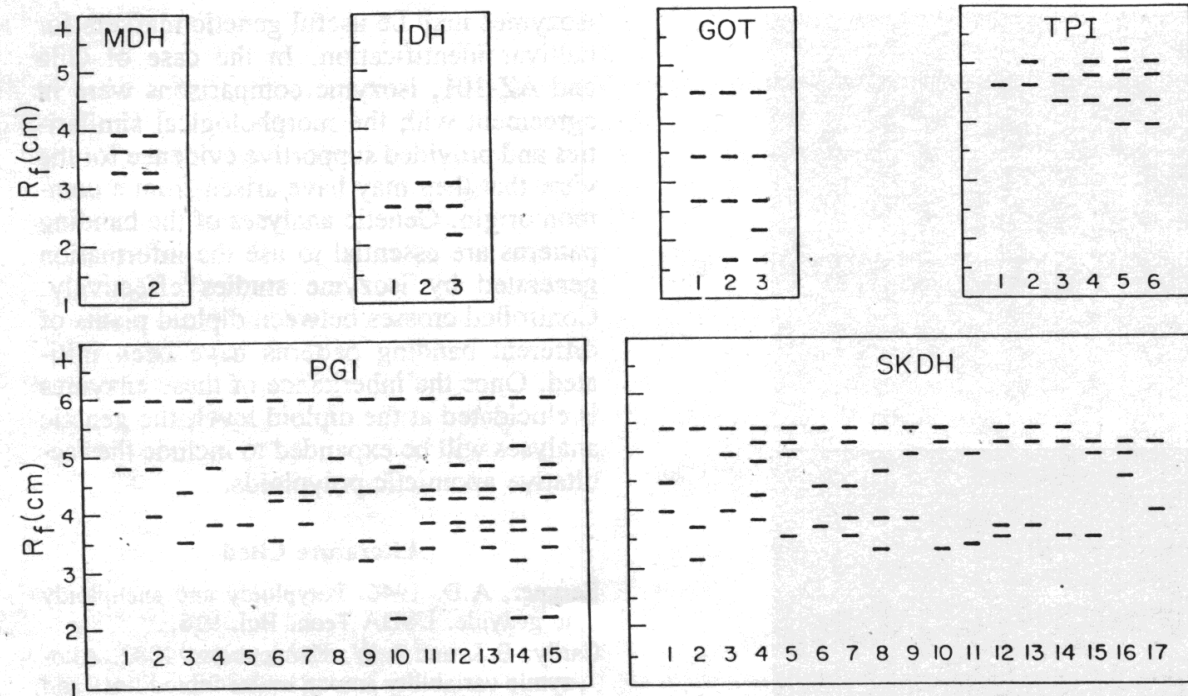

Fig. 1. Schematic illustrations of zymograms showing banding patterns in 47 guayule entries for MDH, IDH, GOT, TPI, PGI, and SKDH.

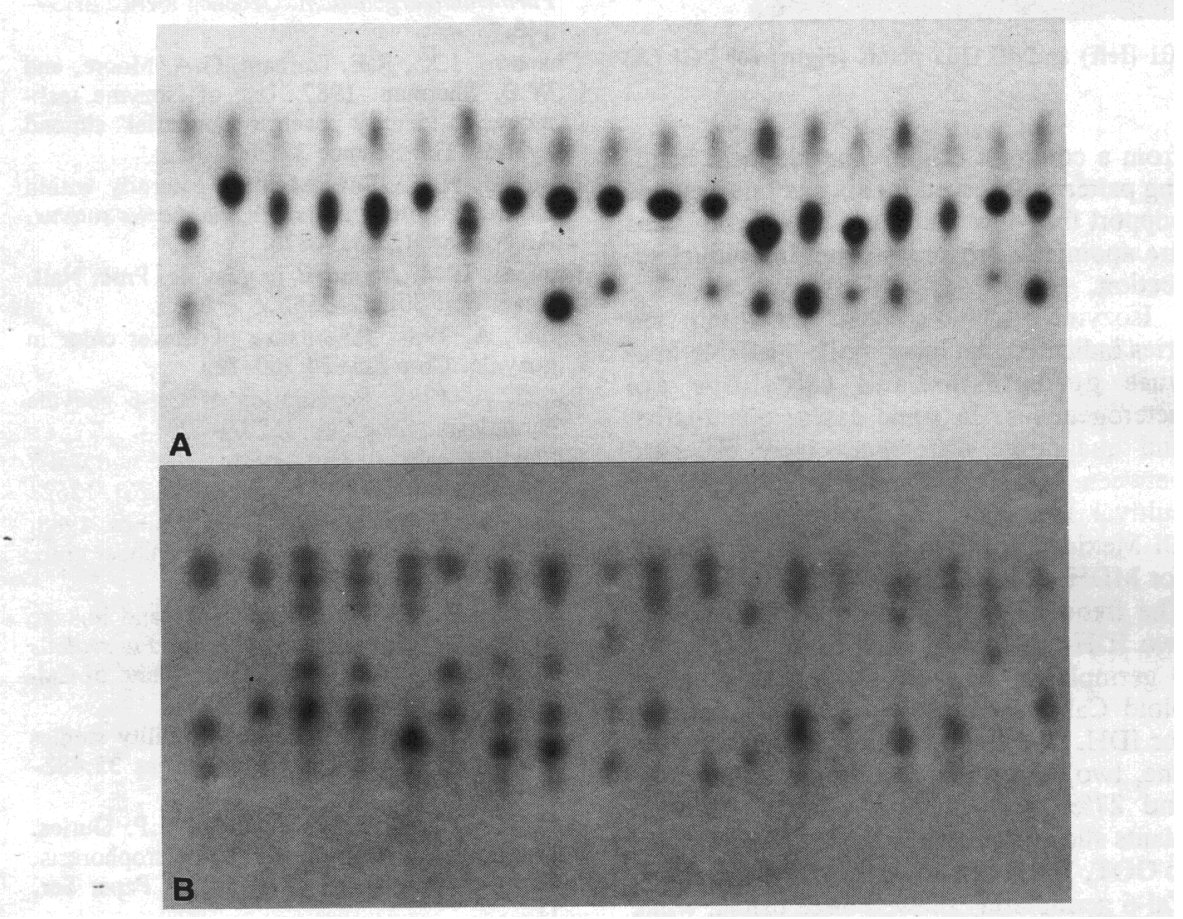

Fig. 2. Variation in PGI banding patterns within Cal-3 (A) and SKDH banding patterns of 10 guayule accessions from Mexico (B).

their banding patterns of 17 enzyme systems and to identify those that provide sufficient information for germplasm identification and genetic studies.

Leaf samples were obtained from 47 guayule entries, including seven USDA cultivars; 23 new polyploid selections of ours; Cal-3, Cal-6, and Cal-7 germplasms; 12 accessions collected near Mapimi in Durango, Mexico; and two other germplasms commonly known as AZ-101 and Gila. Seeds of the last two germplasms were received from D.D. Rubis (Univ. of Arizona) and M. Mittleman (Amerind Agrotech Laboratories, Gila River Indian Community, Ariz.). All entries were grown in a field at the Univ. of California, Riverside. AZ-101 and Gila were represented by 25 plants each. At least 10 plants were sampled for each of the other entries. In this paper, the term germplasm refers to heterogenous populations from cross pollination of sexual diploids, open pollination of facultatively apomictic polyploid plants. or interspecific hybridization.

The enzymes assayed were aconitase (ACN, EC 4.2.1.3), alcohol dehydrogenase (ADH, EC 1.1.1.1), aldolase (ALD, EC 4.1.2.13), catalase (CAT, EC 1.11.1.6), esterase (EST, EC 3.1.1.1), formic acid dehydrogenase (FDH, EC 1.2.1.1), GOT, IDH, leucine aminopeptidase (LAP, EC 3.4.11.1), $\mathrm{MDH}$, malic enzyme (ME, EC 1.1.1.40), menadione reductase (MR, EC 1.6.99.2), PGI, phosphoglucomutase (PGM, EC 2.7.5.1), 6-phosphogluconate dehydrogenase (6PGDH, EC 1.1.1.44), SKDH, and TPI. Only GOT, IDH, MDH, PGI, SKDH, and TPI produced recognizable and repeatable banding patterns for leaf extracts.

Fresh leaf samples were obtained from the field in the morning and brought to the laboratory over crushed ice. About $2.5 \mathrm{mg}$ leaf per plant was placed in plexiglass wells that were kept cool with crushed ice. Each sample was thoroughly macerated in a well with the coarse end of a glass rod using $0.1 \mathrm{ml}$ of cold extraction buffer. The extraction buffer consisted of $0.1 \mathrm{~m}$ Tris- $\mathrm{HCl}(\mathrm{pH} 8.0), 0.005$ M dithiothreitol, $0.2 \%$ 2-mercaptoethanol, $0.001 \mathrm{~m}$ EDTA (tetrasodium salt), $0.01 \mathrm{M}$ potassium chloride, $0.01 \mathrm{M}$ magnesium chloride hexahydrate, and $15 \%$ soluble polyvinylpyrolidone (PVP 40,000).

Three starch gel systems were used in this study. In the morpholine citrate (MC) system, the gel consisted of $9.6 \%$ starch and $0.0016 \mathrm{M}$ citric acid and the electrode buffer consisted of $0.04 \mathrm{M}$ citric acid. Both buffers were titrated to $\mathrm{pH} 7.0$ by the addition of $\mathrm{N}$ (3-aminopropyl)-morpholine (O'Malley et al., 1980). MDH and IDH were studied with the MC system. In the histidine citrate system (HC), the gel consisted of $9.6 \%$ starch and 0.009 M L-histidine titrated to $\mathrm{pH} 6.0$ with citric acid. The electrode buffer was 0.065 M L-histidine (Ellstrand, 1984). PGI and SKDH were assayed with the HC system. In the Tris EDTA boric acid (TEB) system, the gel consisted of $0.045 \mathrm{~m}$ Tris, $0.001 \mathrm{~m}$ EDTA, $0.025 \mathrm{M}$ boric acid, and $12 \%$ starch. The electrode buffer consisted of 0.149 M Tris, 0.0033 м EDTA, and $0.0828 \mathrm{~m}$ boric acid (Ruddle and Nichols, 1971). GOT and TPI were run using the TEB system.

Wicks $1 \times 10 \mathrm{~mm}$ were placed in the wells to absorb the extracts. The wicks were then removed, lightly blotted, and loaded into a transverse slice cut in the starch gels. Electrophoresis was conducted in an incubator at $4 C$, with a tray of ice placed on each gel. Duration, current, and voltage for electrophoresis varied with the gel systems. The $\mathrm{MC}$ and $\mathrm{HC}$ gels were run at $150 \mathrm{~V}$ for $5 \mathrm{hr}$ at 30 mamp and $5.5 \mathrm{hr}$ at 25 mamp, respectively. The TEB gels were run for $4.5 \mathrm{hr}$ at 50 mamp and $200 \mathrm{~V}$. After the electrophoretic run, the gels were sliced into four slabs, all of which were stained.

The staining mixtures for IDH, PGI, and SKDH have been reported (Garvin, 1987). The procedures of Soltis et al. (1983) were used for staining MDH, GOT, and TPI with the following modifications: For MDH, 13.4 dl-malic acid and $10.7 \mathrm{~g}$ of sodium carbonate were dissolved separately in $50 \mathrm{ml}$ of deionized $\mathrm{H}_{2} \mathrm{O}$. Sodium carbonate was slowly added to malic acid over an ice bath and the $\mathrm{pH}$ was adjusted to 8.0 with $\mathrm{NaOH}$. For staining, $5 \mathrm{ml}$ of the above substrate was mixed with $20 \mathrm{ml}$ Tris. $\mathrm{HCl}(0.2 \mathrm{M}$, $\mathrm{pH} 8.0)$, $20 \mathrm{ml} \mathrm{H}$ O, $10 \mathrm{mg}$ NAD, $7.5 \mathrm{mg} \mathrm{NBT}$, and $2 \mathrm{mg}$ PMS. For GOT, the mixture included 


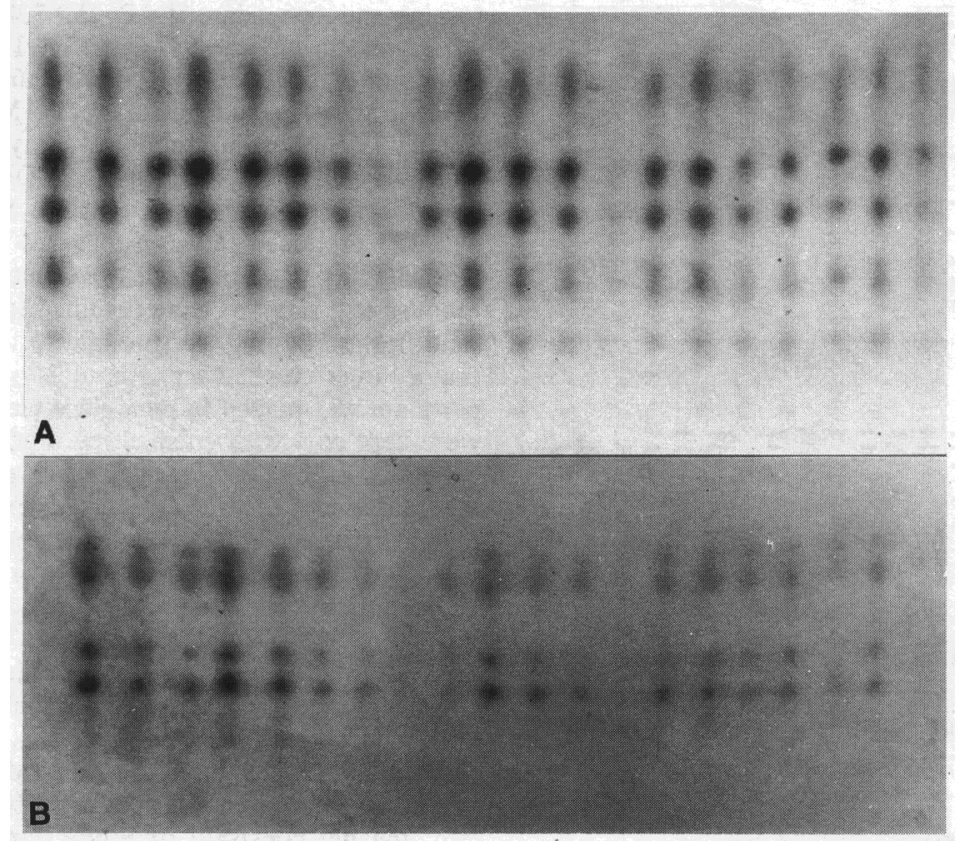

Fig. 3. Similarities in banding patterns of 10 AZ-101 (left) and 10 Gila plants (right) for PGI (A) and SKDH (B).

$25 \mathrm{ml}$ Tris $\cdot \mathrm{HCl}, 25 \mathrm{ml} \mathrm{H} \mathrm{O}_{2}, 100 \mathrm{mg}$ L-aspartic acid, $30 \mathrm{mg} \alpha$-ketoglutaric acid, $5 \mathrm{mg}$ pyroxidal 5-phosphate, and $50 \mathrm{mg}$ fast blue BB salt. The mixture for TPI contained 25 $\mathrm{ml}$ Tris $\cdot \mathrm{HCl}, 15 \mathrm{ml} \mathrm{H} \mathrm{O}, 19 \mathrm{mg}$ EDTA, 100 $\mathrm{mg}$ arsenic acid, $5 \mathrm{mg}$ dihydroxy-acetone phosphate, 100 units glyceraldehyde-3 phosphate dehydrogenase, $5 \mathrm{mg}$ NAD, $1 \mathrm{mg}$ PMS, and $2.5 \mathrm{mg}$ MTT. All gels were stained in the dark until the bands appeared. Gels assayed for GOT were stained at room temperature $(\approx 25 \mathrm{C})$. All other enzymes were assayed at $35 \mathrm{C}$. The stained GOT gels were fixed in $50 \%$ glycerole. Stained gels of other enzymes were fixed in 50\% methanol.

Polymorphisms were observed for all six enzymes (Fig. 1). Considering all entries, MDH, IDH, GOT, TPI, PGI, and SKDH showed two, three, three, six, 15, and 17 distinct banding patterns, respectively. Figure $2 \mathrm{~A}$ shows the variation within $\mathrm{Cal}-3$ germplasm for PGI. Differences between 10 Mexican accessions for SKDH are shown in Fig. 2B.

Plants of AZ-101 and Gila were homogeneous for all six enzyme systems. This result agrees with the morphological uniformity among plants in each of the two germplasms. In addition, AZ-101 and Gila showed identical banding patterns for every enzyme studied. The similarities for PGI and SKHD are presented in Fig. 3. AZ-101 is the progeny of a single plant selection (D. D. Rubis, personal communication). It is a vigorous, high-biomass producer, and highly apomictic. The origin of Gila, on the other hand, is not known. Because of its morphological similarity to AZ-101, however, it is assumed that Gila and AZ-101 were derived from a common origin. The identical banding patterns observed for six enzyme systems support the view that these two germplasms are apomictic progenies of an individual selection.

Isozyme variations observed within entries indicated that most of the available guayule germplasms and selections are heterogeneous. In some cases, quantitative and qualitative differences were observed between entries that may prove useful for cultivar identification. For example, while all Mexican accessions showed three bands for MDH, AZ-101 and Gila had four bands. The frequencies of plants showing one and two IDH bands were $38 \%$ and $62 \%$ in Cal3 germplasm, respectively. Plants of tetraploid Cal-6 showed three banding patterns for IDH. The frequencies of plants showing one, two, and three bands were $33 \%, 40 \%$, and $27 \%$, respectively. AZ-101 and Gila plants showed two IDH bands. With respect to GOT, $100 \%$ of individuals from Cal-3 and Cal-6 germplasms showed three bands, while AZ-101 and Gila showed five bands. The frequencies of plants showing three and four bands were $80 \%$ and $20 \%$, respectively, for one of the Mexican accessions. All entries had individuals showing only one TPI band. The frequency of those plants, however, varied from $5 \%$ in one of the Mexican accessions to $100 \%$ in AZ-101 and Gila. Cal-3 plants had one or two TPI bands with the frequency of $89 \%$ and $11 \%$, respectively, and Cal-6 consisted of individuals with one, two, and three bands with the frequency of $75 \%$, $15 \%$, and $10 \%$, respectively.

Our study revealed that isozyme polymorphism is prevalent in guayule and that isozymes may be useful genetic markers for cultivar identification. In the case of Gila and AZ-101, isozyme comparisons were in agreement with the morphological similarities and provided supportive evidence for the view that they may have arisen from a common origin. Genetic analyses of the banding patterns are essential to use the information generated by isozyme studies effectively. Controlled crosses between diploid plants of different banding patterns have been initiated. Once the inheritance of these enzymes is elucidated at the diploid level, the genetic analyses will be expanded to include the facultative apomictic polyploids.

\section{Literature Cited}

Bergner, A.D. 1946. Polyploidy and aneuploidy in guayule. USDA Tech. Bul. 918.

Cardy, B.J. and L.W. Kannenberg. 1982. Allozymic variability among maize inbred lines and hybrids. Application for cultivar identification. Crop Sci. 22:1016-1020.

Catcheside, D.G. 1950. The B-chromosomes of Parthenium argentatum. Genetica Iberica 2:139148.

Chaparro, J.X., R.E. Durham, G.A. Moore, and W.B. Sherman. 1987. Use of isozyme techniques to identify peach $\times$ 'nonpariel' almond hybrids. HortScience 22:300-302.

Ellstrand, N.C. 1984. Multiple paternity within the fruits of the wild radish, Raphanus sativus. Amer. Nat. 123:819-828.

Esau, K. 1944. Apomixis in guayule. Proc. Natl. Acad. Sci. 30:352-355

Estilai, A. 1984. Inheritance of flower color in guayule. Crop Sci. 24:760-762.

Estilai, A. 1985. Registration of Cal-5 guayule germplasm. Crop Sci. 25:369-370.

Estilai, A. 1986. Registration of Cal-6 and Cal-7 guayule germplasm. Crop Sci. 26:1261-1262.

Estilai, A., H.H. Naqvi, and J.G. Waines. 1988. Developing guayule as a domestic rubber crop. Calif. Agr. 42:29-30.

Garvin, D.F. 1987. The inheritance and linkage of isozyme genes in tepary bean, Phaseolous acutifolius. A. Gray, MS Thesis, Univ. of California, Riverside.

Gerstel, D.U. 1950. Self-incompatibility studies in guayule: II. Inheritance. Genetics 35:482506.

O'Malley, D., N.C. Wheeler, and R.P. Guries. 1980. A manual for starch gel electrophoresis. Univ. of Wis. Dept. of For. Staff Paper Ser. 11 .

Ruddle, F.H. and E.A. Nichols. 1971. Starch gel electrophoretic phenotypes of mouse $\mathrm{x}$ human somatic cell hybrids and mouse isozyme polymorphism. In Vitro 7:120-131.

Soltis, D.E., C.H. Haufler, D.C. Darrow, and G.J. Gastony. 1983. Starch gel electrophoresis of fern: A compilation of grinding buffers, gel and electrode buffers, and staining schedules. Amer. Fern J. 73:9-27.

Tysdal, H.M., A. Estilai, I.A. Siddiqui, and P.F. Knowels. 1983. Registration of four guayule germplasms. Crop Sci. 23:189.

Zaiger, J.K., D.E. Soltis, and G.K. Brown. 1984. Electrophoretic evaluation of registered Parthenium argentatum germplasm Cal-3. El Guayulero 6(3):9-12. 